\title{
Bright High-Harmonic Generation through Coherent Synchrotron Emission Based on the Polarization Gating Scheme
}

\author{
Chuliang Zhou $\mathbb{D}^{1,2}$ Ye Tian $\mathbb{D}^{1,}{ }^{1,2}$ Yushan Zeng $\mathbb{D}^{1,2}$ Zhinan Zeng $\mathbb{D}^{1,2}$ and Ruxin Li $\mathbb{D}^{1,2,3}$ \\ ${ }^{1}$ State Key Laboratory of High Field Laser Physics and CAS Center for Excellence in Ultra-intense Laser Science, \\ Shanghai Institute of Optics and Fine Mechanics, Chinese Academy of Sciences, Shanghai 201800, China \\ ${ }^{2}$ Center of Materials Science and Optoelectronics Engineering, University of Chinese Academy of Sciences, Beijing 100049, China \\ ${ }^{3}$ School of Physical Science and Technology, ShanghaiTech University, Shanghai 201210, China \\ Correspondence should be addressed to Ye Tian; tianye@siom.ac.cn and Zhinan Zeng; zhinan_zeng@siom.ac.cn
}

Received 13 October 2021; Revised 23 December 2021; Accepted 26 January 2022; Published 14 February 2022

Academic Editor: Bhuvanesh Ramakrishna

Copyright (c) 2022 Chuliang Zhou et al. This is an open access article distributed under the Creative Commons Attribution License, which permits unrestricted use, distribution, and reproduction in any medium, provided the original work is properly cited.

\begin{abstract}
Relativistic surface high harmonics, combined with the use of polarization gating, present a promising route towards intense single attosecond pulses. However, they impose stringent requirements on ultra-high laser contrast and are restricted by large intensity losses in real experiments. Here, we numerically demonstrate that by setting an optimal time delay in the polarization gating scheme, the intensity of the generated single attosecond pulses can become approximately 100 times stronger than that with nonoptimal time delay in the coherent synchrotron emission process. When a petawatt-class driving laser irradiates a solid target, an ultra-dense electron nanobunch and a strong space-charge sheath develop, and the accumulated electrostatic energy is only released in half of the laser cycle when this electron nanobunch moves backward. This process results in the emission of intense high harmonics. Our study provides a reliable method for developing bright attosecond extreme ultraviolet pulses.
\end{abstract}

\section{Introduction}

High-harmonic generation ( $\mathrm{HHG})$ from an ultra-intense laser solid-target interaction has been proven to be an important method for producing bright coherent extreme ultraviolet rays and X-rays [1-3]. Generation of attosecond pulse trains on the solid surface has also recently been demonstrated experimentally $[4,5]$. Moreover, the coherent wake emission (CWE) $[3,4,6,7]$ and relativistic oscillating mirror (ROM) [1, 8-14] models successfully describe the high-harmonics generation process at laser intensities of $I<10^{18} \mathrm{~W} / \mathrm{cm}^{2}$ and $I>10^{18} \mathrm{~W} / \mathrm{cm}^{2}$, respectively. In recent years, nanobunching of relativistic electrons has been identified to be responsible for coherent synchrotron emission (CSE) [15-18]. An alternative theory of relativistic electronic springs (RESs) has also been proposed [19]. In the CSE model, dense nanometer-scale bunches of electrons accelerated by the laser and plasma fields in relativistic trajectories radiate high-frequency light. The most widely investigated scheme is the ROM mechanism, because of the relativistic Doppler upshift. Light reflected from a periodic oscillating mirror comprises a broad harmonic spectrum. For near-normal incidence, the plasma oscillations are driven by the Lorentz force of the incident laser pulse and charge-separation-induced electrostatic fields. For a laser pulse with linear polarization, the Lorentz force varies as $F_{p} \sim a^{2}(t) \cdot\left[1+\sin \left(2 \omega_{L} t\right)\right], \quad$ where $a^{2}(t)=I_{L}(t) \cdot \lambda_{L}{ }^{2} /$ $\left(1.37 \times 10^{18} \mathrm{~W} \mu \mathrm{m}^{2} \mathrm{~cm}^{-2}\right)$ is the normalized vector potential. In the case of the circularly polarized pulse, this force is of the form $F_{p} \sim a^{2}(t)$. Thus, it exhibits no fast oscillations, creating a smooth density depression, with which no harmonics is allowed to generate.

The critical requirement for effective harmonic generation in high-intensity laser systems is the effective control over the purity of the laser pulses to avoid any effect of the pedestal on the target. It has been verified that the most efficient HHG happens when an optimal preplasma scale length $L_{s}\left(0.05 \lambda_{L}<L_{s}<0.2 \lambda_{L}\right)$ is introduced [3, 20, 21]. In this case, an ultra-high laser contrast, usually higher than $10^{-10}$, will be required, which poses formidable experimental 
challenges to current high power laser facilities [22]. Hence, a feasible method should be found to relax the severe requirement on the preplasma scale length.

Conventionally, the existence of a long scale, nonsteplike preplasma in front of the target would significantly decrease the coherence and conversion efficiency of the harmonics. To avoid such detrimental effects and obtain single attosecond pulses, one can use few-cycle laser pulses with carrier-envelope phase stabilization or alternatively use longer pulses with polarization gating, a well-known technique commonly applied in gas-phase HHG. However, in the pioneering work on polarization gating for plasma HHG [23], the density gradient scale length at the plasma-vacuum interface was not taken into consideration, which would have a significant effect on the plasma HHG.

Different polarization gating techniques have been proposed for many-cycle laser pulses that suffer from large intensity losses [23-25]. Recently, noncollinear polarization gating [25] has been demonstrated, which is simpler and more practical than the conventional polarization gating $[23,24]$. For a given gate, the amplitude in the linear region of the pulse is smaller when using a longer pulse duration for polarization gating. However, a large fraction of the energy cannot be exploited in the circular polarization part.

In this study, we discuss the generation of intense high harmonics using multicycle driving pulses, in combination with the polarization gating scheme (Figure 1), and even under nonoptimal plasma-scale-length conditions. The efficiency of HHG from laser-plasma interaction can be boosted by exploiting a polarization gating scheme, which results in a recession of the electron in preplasma due to the pressure of the laser. Subsequently, an ultra-dense electron bunch and a strong electrostatic potential are formed, resulting in strong CSE. It is verified that the polarization gating technique driven by a multicycle laser can be used to develop an intense extreme ultraviolet source effectively in laser systems with a lower contrast. Our findings can also be applied to noncollinear polarization gating [25]. In the noncollinear gating scheme, another noncollinear gating term that arises from the wavefront rotation due to the noncollinearity of the two beams will be introduced. But if the angle $\gamma$ that the wave front of each half beam makes with the center axis is small $(\gamma<<1)$, the effect of wavefront rotation can be ignored.

\section{Methods}

Polarization gating $[23,26,27]$ is created by combining two same-color, counter-rotating, and circularly polarized multicycle Gaussian laser pulses with a certain time delay between them, as shown in Figure 1(a). This pulse possesses a time-dependent ellipticity. A gate of nearly linear polarization is opened when the two pulses have similar intensities. The peak field amplitude $E_{0}$, carrier frequency $\omega$, fullwidth at half-maximum (FWHM) pulse duration $\tau$, and carrier-envelope phase $\varphi$ are identical for the two pulses, where the time delay between them is $T_{d}$. The electric fields of the left and right circularly polarized pulses propagating in the $x$-direction are, respectively,

$$
\begin{aligned}
\vec{E}_{l}= & E_{0} \exp \left(-2 \ln 2\left(\frac{t}{\tau}\right)^{2}\right) \\
& \cdot[\vec{y} \cos (\omega t+\varphi)+\vec{z} \sin (\omega t+\varphi)], \\
\overrightarrow{E_{r}}= & E_{0} \exp \left(-2 \ln 2\left(\frac{t-T_{d}}{\tau}\right)^{2}\right) \\
& \cdot[\vec{y} \cos (\omega t+\varphi)-\vec{z} \sin (\omega t+\varphi)] .
\end{aligned}
$$

The combined electric field is $E(t)=\overrightarrow{E_{l}}+\overrightarrow{E_{r}}$. The ellipticity $\xi(t)$ is defined as the ratio of the minor and major axes of the electric field vector ellipse, $\xi(t)=\left|E_{y}\right| /\left|E_{z}\right|$, and the evolution of ellipticity with time is given by [23]

$$
\xi(t)=\exp \left(-\frac{4 \ln 2 \times \mid\left(t-T_{d}\right)^{2}-t^{2}}{\tau^{2}}\right) .
$$

For a threshold ellipticity value of 0.4 , the gating time is given by

$$
\delta_{\text {gating }}=\frac{0.2 \tau^{2}}{T_{d}} .
$$

The ellipticity evolution in polarization gating with different relative time delays is shown in Figure 2(a). When $T_{d}$ is close to the pulse duration, the gating time is about one laser cycle, the isolated attosecond pulse can be obtained by filtering out the low frequency. The relative delay will affect the gate width, the intensity, and the degree of polarization of the linearly polarized laser in the gate width. We performed particle-in-cell (PIC) simulations with the kinetic code EPOCH [28] to study harmonic generation in relativistic laser interaction with overdense plasma. Two counter-rotating CP Gaussian pulses (its normalized intensity is $\left.a=e E_{0} / m_{e} c \omega_{0}\right)$ were used as the driving pulses with frequencies of $\omega_{0}\left(\lambda_{L}=800 \mathrm{~nm}\right)$ and copropagated along the $x$-direction. The two pulses both had a FWHM duration $\tau$ of $6 T_{0}$, where $T_{0}=\lambda_{L} / c$ is the laser period and $c$ is the velocity of light in vacuum. When the time delay between them is 0 , the linearly polarized laser is formed. In the following simulation, the two circularly polarized lasers are each composed of two linearly polarized laser pulses with equal intensity but different polarization: one is polarized orthogonally to the other with a $\pi / 2$ phase shift such that their superposition can form a circularly polarized pulse. Therefore, under different time delays, the total energy of the incident laser remains unchanged. The solid target is considered fully ionized, which consists of a $2 \lambda_{L}$ thick slab with $n_{\max }=100 n_{c}$ and an exponentially declining preplasma with a scale length of $L_{s}$, where $n_{\mathrm{c}}=\varepsilon_{0} m_{e} \omega_{0}{ }^{2} / e^{2}$ represents the critical density, and $m_{e}$ and $e$ are the electron rest mass and charge, respectively. The laser duration and plasma density were the same for both one-dimensional (1D) and two-dimensional (2D) simulations. For the $1 \mathrm{D}$ simulation, the grid size was $\mathrm{d} x=\lambda_{L} / 2000$, where each cell contained 1000 macroparticles. The ions were treated as a neutralizing immobile background. While for the $2 \mathrm{D}$ simulation, the grid size was $\mathrm{d} x=\mathrm{d} y=\lambda_{L} / 800$, and each cell contained 100 macroparticles. The reflected emission spectra were determined from the Fourier 


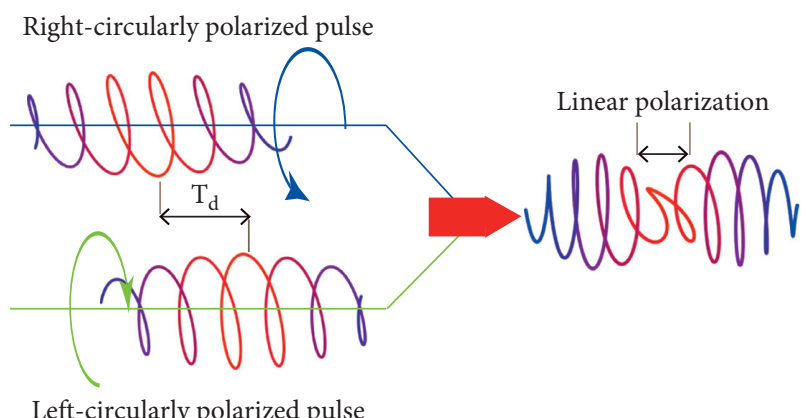

Left-circularly polarized pulse

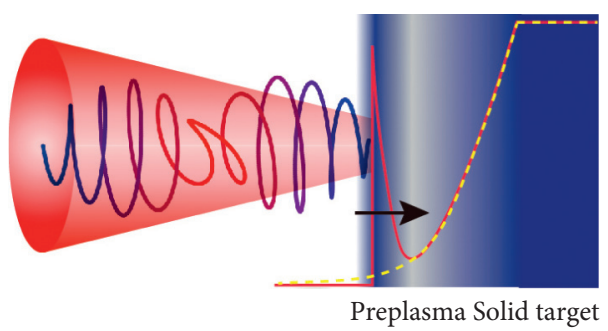

(b)

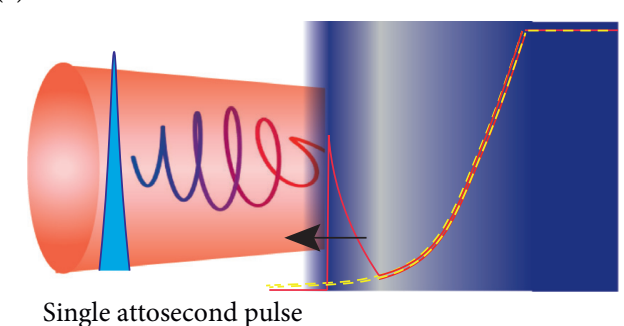

(c)

FIGURE 1: (a) Schematic of intense extreme ultraviolet pulse generation with polarization gating scheme, which consists of two counterrotating circularly polarized (CP) laser pulses normally incident on a solid target. These collinear left and right CP laser pulses create a linear gate in the middle portion. (b) Illustration of the electron density profiles when the front CP laser part irradiates the target. The preplasma is compressed and an ultra-dense electron nanobunch is formed. (c) When the linear polarization of the laser reaches the target surface, the dense electron nanobunch is accelerated by the charge-separation and laser electric field to relativistic velocities in a half laser cycle; thus, intense extreme ultraviolet pulses would be emitted.

transform of the time-resolved reflected electric field at the vacuum gap.

\section{Results and Discussion}

We first clarify the electron density modification caused by the ponderomotive force when the rising edge of the laser interacts with the target. Subjected to light pressure, the electrons in the preplasma are pushed towards the target at first, forming an electron-depleted layer. As a result, an electron density sheath is formed at the position of the vacuum-plasma interface, and the electron density can be substantially compressed to several nanometers (Figure 2(b)). During this stage, the longitudinal electric field component grows until the electrostatic force starts to pull back the bunch. The electrons are piled up in a narrow region under the effect of light pressure, and a charge-separation field is formed between the thin layer of ions and electron nanobunches on the target surface. The dependence of the width and number density of the electron bunch on the preplasma scale length and time delay are present in Figures 2(d) and 2(g); as shown, with increasing the preplasma scale length or time delay, the shortest width of the electron nanobunch has been kept around $2 \mathrm{~nm}$, while the electron number density tends to decrease, which is more obvious in Figure 2(d). Accordingly, Figures 2(e) and 2(h) show the variation of efficiency of harmonic generation with preplasma scale length and time delay, respectively. It can be seen that the evolution of harmonic efficiency is consistent with the variation of nanoelectron layer density in
Figures 2(d) and 2(g). Figure 2(j) shows the dependences of width and number density of the electron nanobunch on the intensity of incident laser, while Figure 2(k) gives the relation between the efficiency of high-harmonic generation and incident laser intensity. As the laser intensity increases, the number density of electron nanobunch will increase as well, while its shortest width shows yet no significant changes. This is analogous to the scenario of changing the preplasma scale length and time delay, the harmonic generation efficiency will increase as the laser intensity strengthens, which verified again the obvious positive correlation between the harmonic generation efficiency and the number density of electron layer. Hence, we can reasonably conclude that the higher electron density is favorable for coherent and stronger radiation. Owing to the relativistic character of electron motion, the displaced electrons can reemit their energy when the electrons propagate back towards the boundary, thereby compressing the reemission into an attosecond burst. An intense extreme ultraviolet pulse is emitted when the linear polarization of the incident laser reaches the target surface.

Figure 2(c) presents the temporal shape of the pulse intensity after passing a bandpass (30th 100th) filter. The number of gated attosecond pulses (only the pulse intensity that is higher than the $1 / e^{2}$ of peak intensity is counted, which can represent the isolation degree) and the corresponding peak intensity for varied preplasma scale length, delays, and incident laser intensity are respectively presented in Figures 2(f)-2(l). From the results displayed in Figure 2(f), the number of attosecond pulses enhances with 


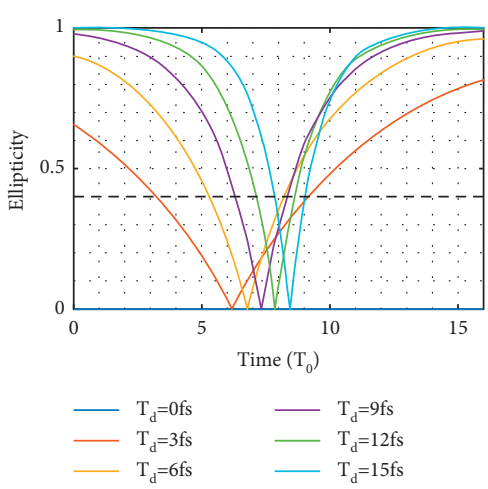

(a)

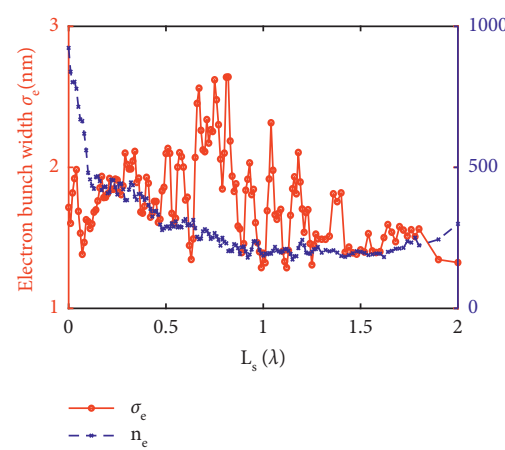

(d)

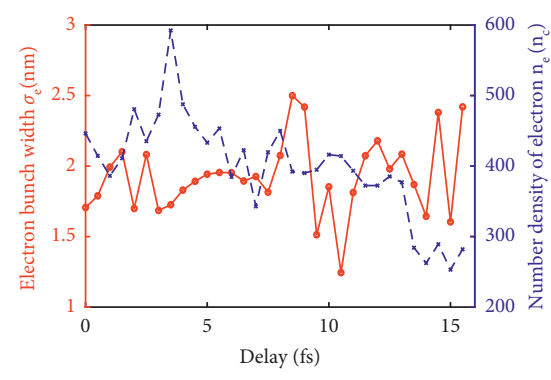

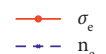

(g)

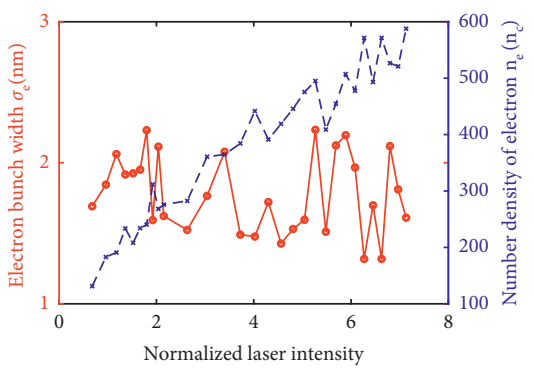

$\because \sigma_{e}$

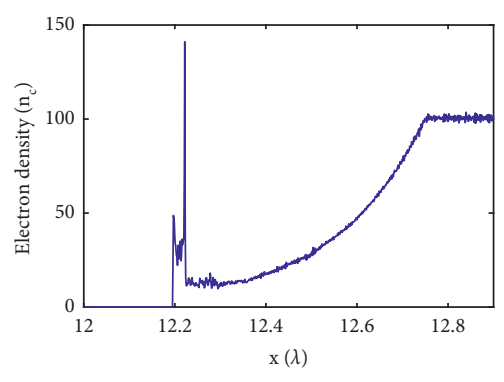

— $\mathrm{Ls}=0.2 \lambda$

(b)

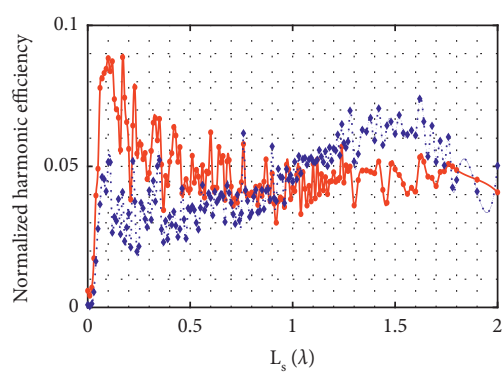

- 10th-20th

20th-100th

(e)

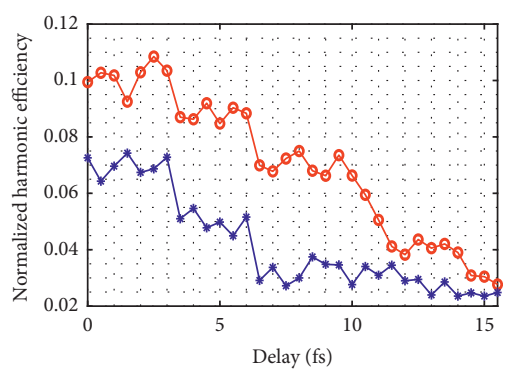

$\rightarrow$ 10th-20th

$\rightarrow$ 20th-100th

(h)

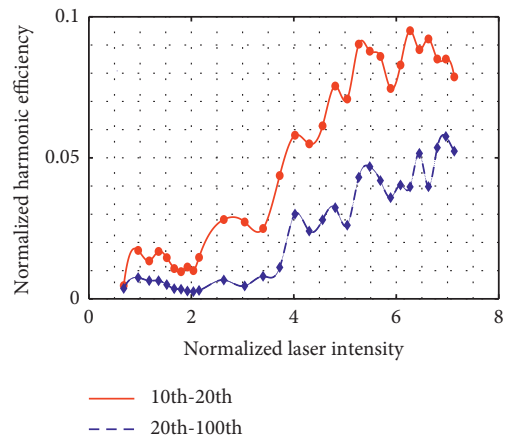

(k)

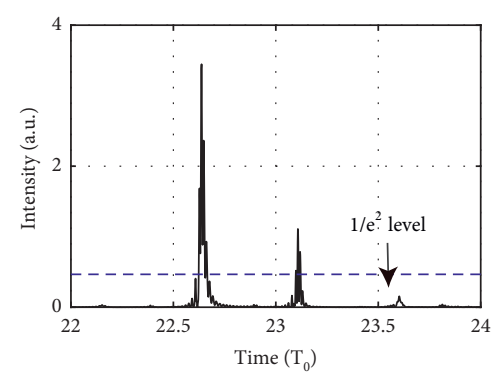

(c)

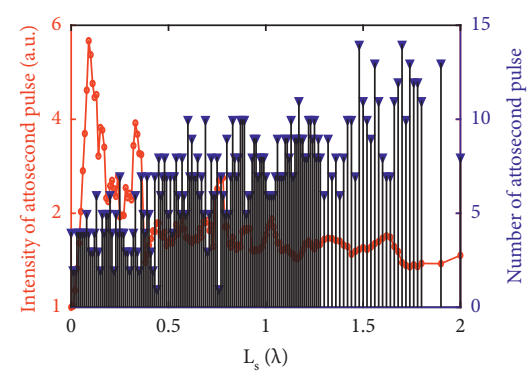

$\because$ Intensity
$\because \quad$ Number

(f)

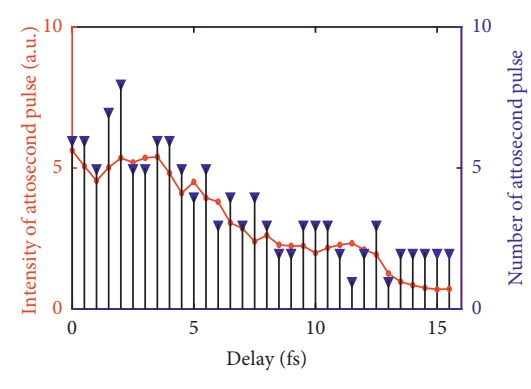

$\rightarrow$ Intensity

(i)

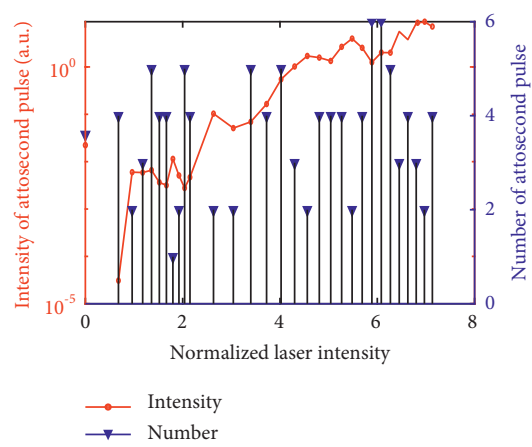

(l)

Figure 2: (a) Evolution of the ellipticity in polarization gating with different relative time delays. (b) Electron density $\left(n_{e}\right)$ right before the generation of the most intense attosecond pulse. PIC simulation parameters: $a=10$, time delay $T_{d}=16 f s$, and Ls $=0.2 \lambda_{L}$. (c) Obtained radiation pulse intensity by applying a spectral filter to select the $30^{\text {th }}$ to $100^{\text {th }}$ harmonic orders. PIC simulation parameters: $a=10$, time delay $T_{d}=12 f s$, and $L s=0.1 \lambda_{L}$. (d, g, j) Dependence of the shortest width and number density of the electron bunch on the preplasma scale length, time delay, and laser intensity. (e, h, k) Dependence of efficiency of harmonic generation on preplasma scale length, time delay, and laser intensity. ( $f, i, 1)$ The dependence of number and the peak intensity of gated attosecond pulses (30th-100th) on the preplasma scale length, time delay, and laser intensity. Simulation parameters (d, e, f) $a=10$, time delay $T_{d}=6 f s ;(\mathrm{g}, \mathrm{h}, \mathrm{i}) a=10, L s=0.1 \lambda_{L} ;(\mathrm{j}, \mathrm{k}, \mathrm{l})$ time delay $T_{d}=6 f s, L s=0.1 \lambda_{L}$. 
increasing preplasma scale length, while a shorter preplasma scale length is more beneficial for obtaining isolated attosecond pulses. In Figure 2(i), the number and intensity of attosecond pulses both show a gradual decrease at longer delays (and thus shorter gate times), and for laser intensity, it can be seen in Figure 2(l) that the intensity of gated attosecond pulse traces approximately exponentially with the driving laser intensity, whereas the degree of isolation appears to be slightly less sensitive to laser intensity variation.

The present working scheme we proposed has been checked under different laser-plasma parameters. In Figure 3, we calculated the influence of plasma scale length $L s$ and time delay $T_{d}$ on high-order harmonic emission from 1D PIC simulations. Results for harmonics within the range from 10th to 20th (Figure 3(a)) and from 20th to 100th (Figure 3(b)) are presented. From these results, we can also see that when the preplasma scale length is too long $(L s>0.4$ $\lambda_{L}$ ), the harmonic efficiency will stay low regardless of the relative time delay. But this efficiency would become sensitive to the delay when a mildly long $\left(L s<0.4 \lambda_{L}\right)$ preplasma scale length is assumed. Furthermore, at larger preplasma scale length (beyond $0.2 \lambda_{L}$ ), the optimal delay also seems to increase. From Figures 2(f) and 2(i) and Figures 3(c) and $3(d)$, we can conclude that the shorter preplasma scale length and shorter gate width are both beneficial to the generation of isolated attosecond pulses.

In Figure 4, we compare the PIC simulation results respectively of two distinct time delays: $16 f s$ (where gating width is about one laser cycle) and $T_{d}=0$ (linearly polarized laser), while the other parameters are set to angle of incidence $\theta=0, a=10$, and $L_{s}=0.2 \lambda_{L}$. The corresponding results at these two time delays are shown in the left $(16 f s)$ and right columns $\left(T_{d}=0\right)$ in Figure 4, respectively. Figures 4(a) and 4(d) depict the electric field evolutions of the laser pulses in these two cases, inside which the amplitude of the reflected field exceeds that of the incoming field (for $E_{y}$, it is up to $30 \%$, while for $E_{z}$, it is $90 \%$ ) for the $16 f s$ scenario in Figure 4(a). We know that under different laser intensities, the harmonic generation efficiency is very different, and there is no simple linear correlation between the efficiency of harmonics generation and the intensity of the incident laser. As a result, a significant difference exists in the behavior of $E_{y}$ and $E_{\mathrm{z}}$. This is only the result of a 1D simulation in which the effect of plasma denting cannot be observed in the electric field. A curved surface can also boost incident lasers [29]. This will be more obvious under the longer preplasma scale. The reflected pulse will be spatially focused, resulting in stronger field strength, whose divergence will also increase by certain extent. The attosecond pulse divergence dependents on the denting of the plasma surface which is not invariant over time. However, in our case, we only concern a small number of consecutive pulses gated from the pulse train. Hence, the divergence should not vary significantly. Owing to the relativistic character of electron motion, the displaced electrons are spatially squeezed to a high-density ultra-thin layer (Figure 2(b)), which can reemit their energy when propagating back towards the boundary, and thereby compressing the reemission into an attosecond burst with larger electric field amplitude than the incident light.
The corresponding harmonic spectra are shown in Figures $4(\mathrm{~b})$ and $4(\mathrm{e})$, where the dashed line marks the universal scaling $I \propto \omega^{-8 / 3}$ predicted by Baeva-Gordienko-Pukhov (BGP) [10]. In both cases, the harmonic spectra remain close to the $I \propto \omega^{-8 / 3}$ scaling law, which also implies the existence of zeros in the transverse momenta of plasma surface electrons. There are some fuzzy harmonic spectral structures observable in linear polarization. For the polarization gating scheme, the harmonic spectral structures are more discernible, especially for higher orders. The time-frequency analyses of the HHG are shown in Figures 4(c) and 4(f), where all frequencies are emitted within a fraction of the driving laser period and trains of attosecond pulses are emitted in the polarization gating scheme. But, we failed to obtain isolated attosecond pulses as expected. Thus, an optimal time delay still awaits to be found when a long preplasma is considered. The superposition of phase-locking-attosecond pulses in different periods contribute to the integrated harmonics spectrum. However, the phase between attosecond pulses generated in different periods will inevitably be accompanied by chirp and phase fluctuations, which will degrade the time coherence of attosecond pulses generated in many cycles (Figure 4(f)) than those generated in fewer cycles (Figure 4(c)). Also, the resulting phase-locking spectrum is not as clear as the polarization gating scheme.

Figure 5(a) shows the electron density overlaid with the longitudinal $J_{x}(x, t)$ current density components. The simulation parameters are the same as Figure 4(a). Here, these currents exhibit a periodic characteristic, in the sense that they undergo longitudinal oscillations twice inside each laser period. This apparent regularity emerges from the electron motion that is aperiodic at the single-particle level. Figure 5(b) shows that the harmonic pulses are emitted in the form of an attosecond pulse train, with two attosecond pulses emitted per optical cycle of the laser field. It is efficiently emitted at the moments when the longitudinal momentum of the electrons reaches its maximum. These results suggest that the mechanism is in accordance with the $\gamma$-spike model of the ROM.

The results in Figure 6 are based on 2D PIC simulations, where a same total laser energy is assumed (using the same laser and plasma quantities as in the $1 \mathrm{D}$ simulation, the transverse size of the focal spot (FWHM) is $7.5 \lambda_{L}$ ). In view of the preplasma scale length in the forthcoming experiments (for example, $L s=0.2 \lambda_{L}$ ), we have comprehensively compared the efficiency of harmonic generation and the isolation degree of attosecond pulses under different delays. In all the cases where single attoseconds can be obtained after frequency filtering by selecting the 30th to 100th harmonic orders, we observed a significant increase and modulation of the harmonic spectrum, as shown in Figure 6(a) (red line) with proper time delays $\left(6 f_{s}\right)$. This result reveals complex plasma dynamics that have not been observed in a similar study [23]. Specifically, we found that an optimal time delay exists in polarization gating when a relatively long preplasma is introduced. The spectrum declines more slowly and shows clearer harmonic structures when $T_{d}$ is chosen as $6 f s$ in our case, and the intensity of 


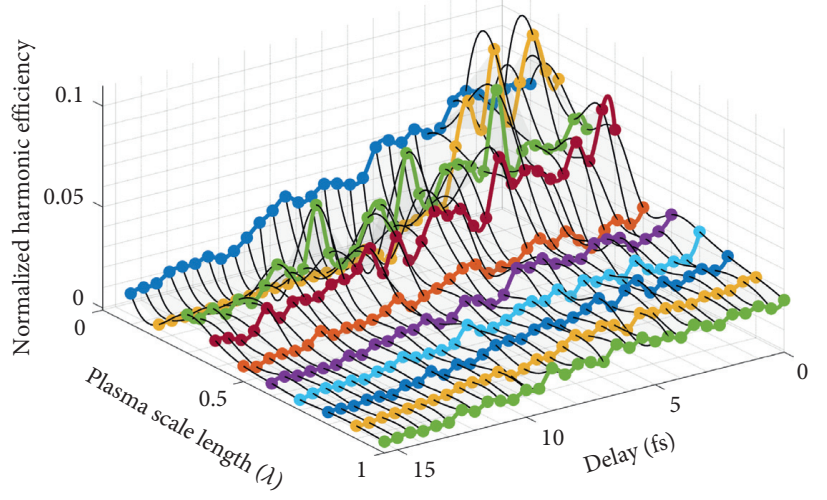

10th-20th harmonics

(a)

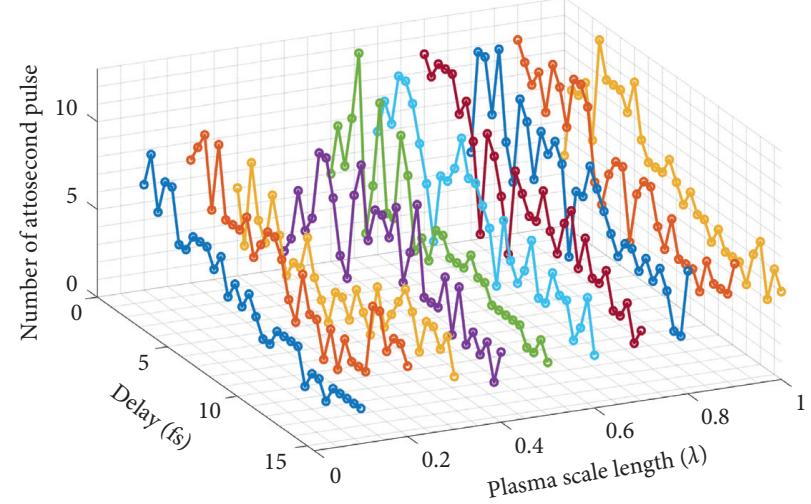

(c)

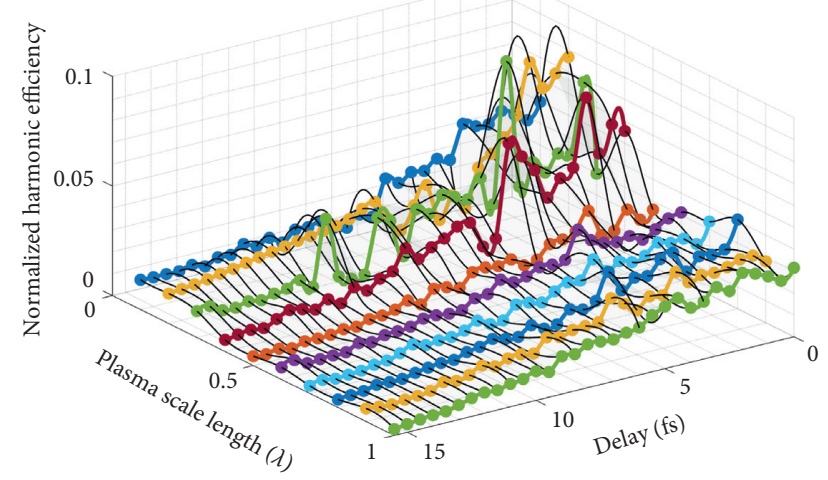

20th-100th harmonics

(b)

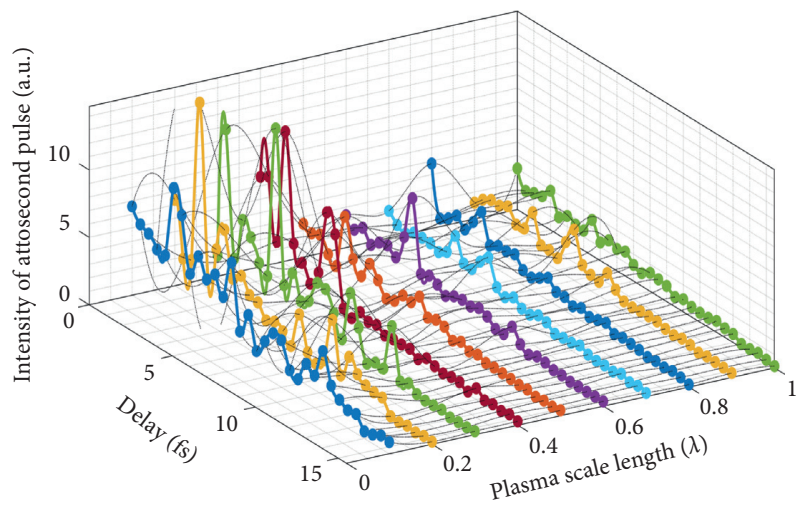

(d)

Figure 3: $(\mathrm{a}, \mathrm{b})$ Harmonics generation efficiency as a function of both plasma scale length and delay of the two circular polarized pulses in polarization gating. (c, d) The number and corresponding peak intensity of gated attosecond pulses (30th-100th) as a function of preplasma scale length and delay between the two circular polarized pulses in the polarization gating scheme. The intensity of the incident field is $a=10$, the pulse duration $\tau$ is $16 f s$, and solid-target density is $100 n_{c}$. Results for harmonics within the range from 10th to 20th (a) and from 20th to 100th (b) are presented.

HHG with optimal time delay can be increased by approximately 100 times when compared with the other nonoptimal time delay (here, the HHG intensity is also presented at $12 f s$ delay, where the gate times are shorter and are generally expected to produce single attosecond pulse more efficiently). The spectrum is very different from the BGP model. Efficient HHG from a relativistic plasma mirror requires (i) the longitudinal electron velocity reaching the maximum and, (ii) meanwhile, the transverse electron momentum reaching the minimum. When the delay is adjusted, the gate width, intensity, and time-varying ellipticity of the laser in the gate width will also be changed. The difference in HHG efficiency between the different delay cases can be attributed to the phase difference and field intensity, which leads to different plasma dynamics. On a macroscopic level, the spectrum is no longer well characterized by a universal decay index $p=8 / 3$, or others. There is now a well-defined break in the spectrum in Figure 6(a), centered at $n=\sim 50$ th. From previous publications [20] and the results of Figure 2(e), the optimal plasma scale length in our case is $\sim 0.1 \lambda_{L}$. Also, from Figure $6(\mathrm{a})$, we can see that there exists an optimal time delay, even at a longer plasma scale length (here, we choose $0.2 \lambda_{L}$ as an example), at which the harmonic structure in the spectrum is still clear and its intensity is also enhanced, suggesting that this process works well at a longer scale length than the typical optimal scale length. Thus, it opens the possibility of relaxing the severe requirement on the preplasma scale length. In the case of a polarization-gated pulse, the surface oscillations are highly suppressed during the circular polarization. The plasma denting effect, as mentioned before, was also observed. In addition, in Figures 6(b) and 6(c), it is observed that a train of attosecond electron bunches is emitted during the interaction. It should originate from the aforementioned electron nanobunch and shows some similarity with the electron bunching process [30] in free-electron lasers.

Figures $7(\mathrm{a})$ and $7(\mathrm{~b})$ present different spatiotemporal evolutions of the electron density, corresponding to the two different delays in Figure 6(a), at $L s=0.2 \lambda_{L}$. It is shown that a more energetic electron bunch is formed and moves toward the incident laser when the $T_{d}$ is $6 f s$. This indicates that the dominant radiation generation is the strong synchrotron emission from the ultra-relativistic electron bunch, which occurs when the compressed ultra-dense nanobunch 

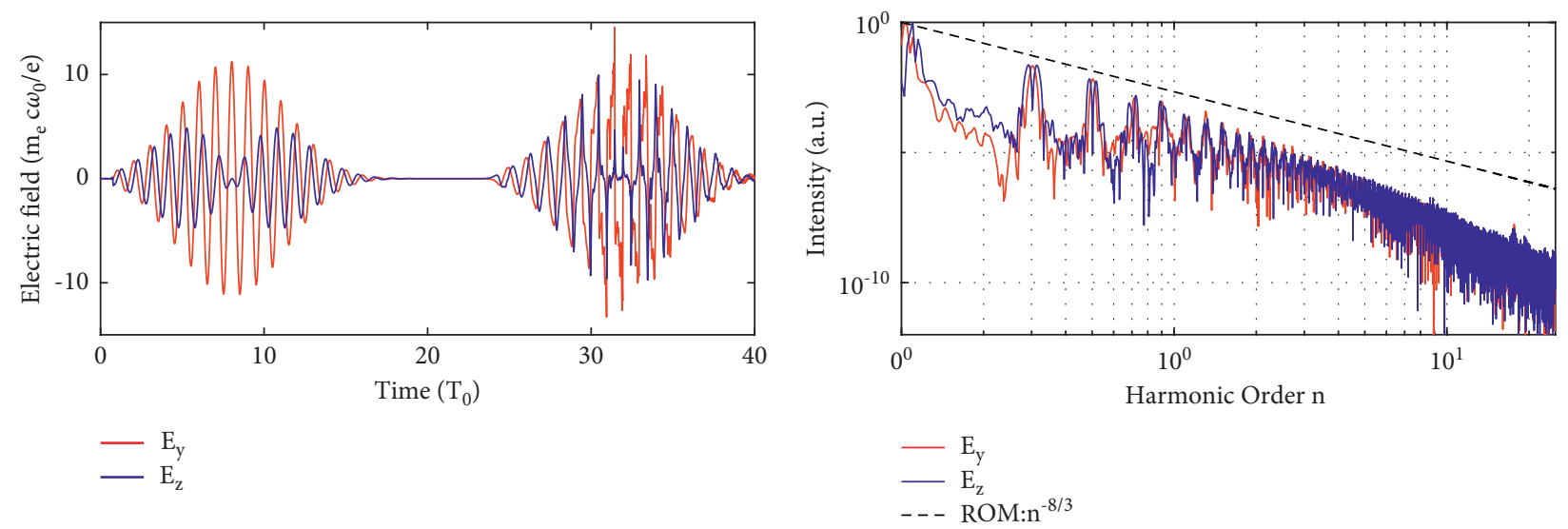

(a)

(b)
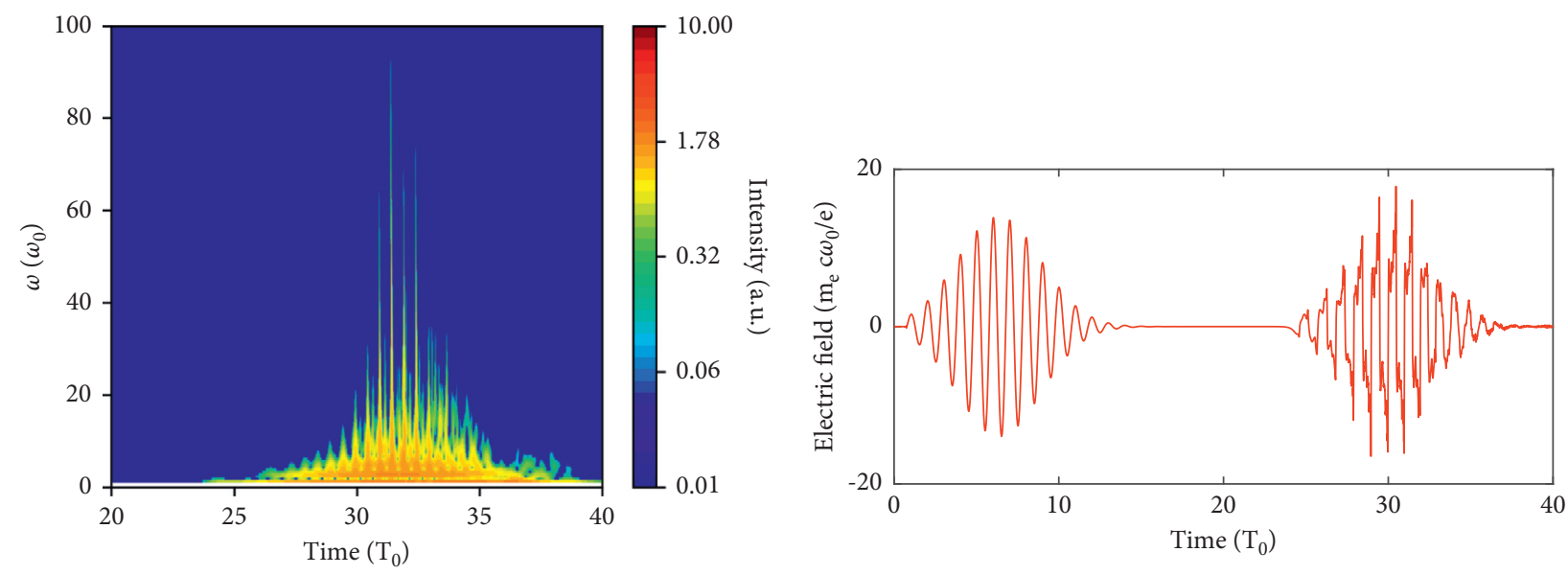

(c)

(d)

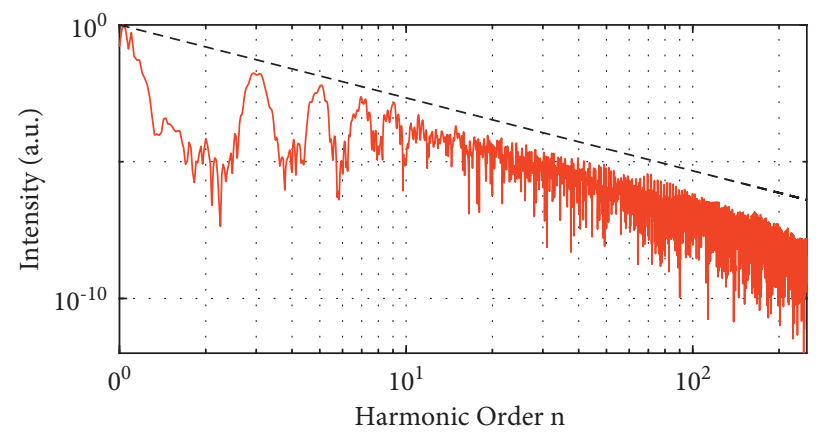

$\mathrm{E}_{\mathrm{y}}$
$--\mathrm{ROM}: \mathrm{n}^{-8 / 3}$

(e)

(f)

FIgURe 4: PIC simulation results for two different time delay cases: $T_{d}=16 f s$ (left panel, originally thought to be suitable for isolated attosecond pulse generation) and $T_{d}=0$ (right panel, corresponding to linear polarized laser), other parameters: $a=10, L_{s}=0.2 \lambda_{L}$, and incidence angle $\theta=0$. (a,d) The incoming and reflected electric fields at a fixed position before the target surface, respectively. (b, e) Fourier spectra of the reflected pulses. The dashed line marks the universal scaling law $I \propto \omega^{-8 / 3}$ predicted based on the Baeva-Gordienko-Pukhov (BGP) theory. (c, f) The time-frequency analyses of the HHG plotted on a logarithmic scale. 


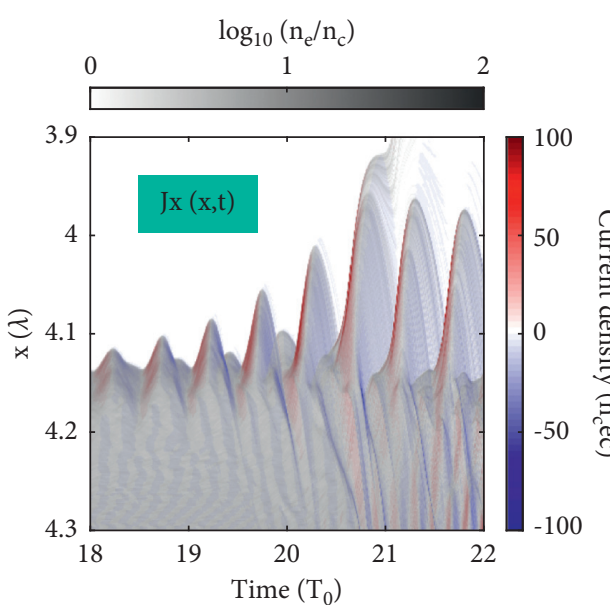

(a)

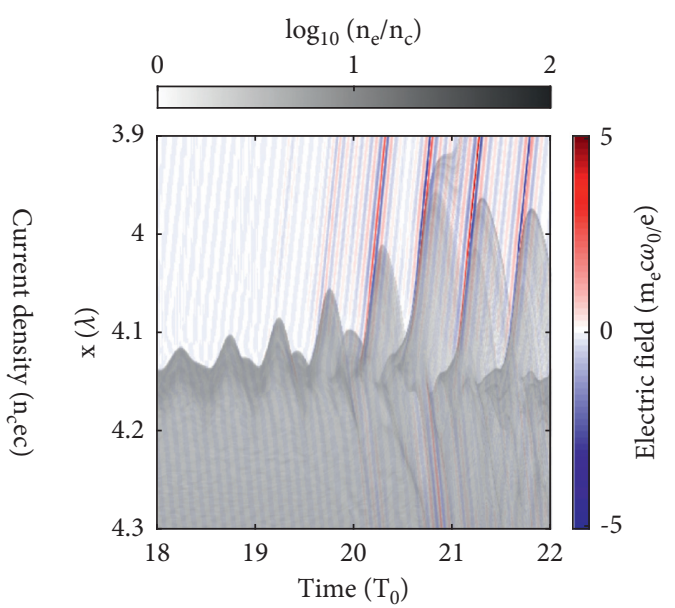

(b)

FIGURE 5: (a) Spatiotemporal density evolution of the electrons overlaid with longitudinal current density $J_{x}(x, t)$ for the polarization gating case. The overall motion of the front surface consists of longitudinal oscillations twice every laser period. (b) Electron density on a logarithmic scale and the emission of harmonic pulse. The harmonic fields are frequency-filtered by $N>10$ th order, showing that the harmonic pulses are emitted in the form of an attosecond pulse train. Radiation is efficiently emitted at the moments when the longitudinal momenta of the electrons reach a maximum, which is also when both transverse momenta of the plasma surface electrons reach zero simultaneously.

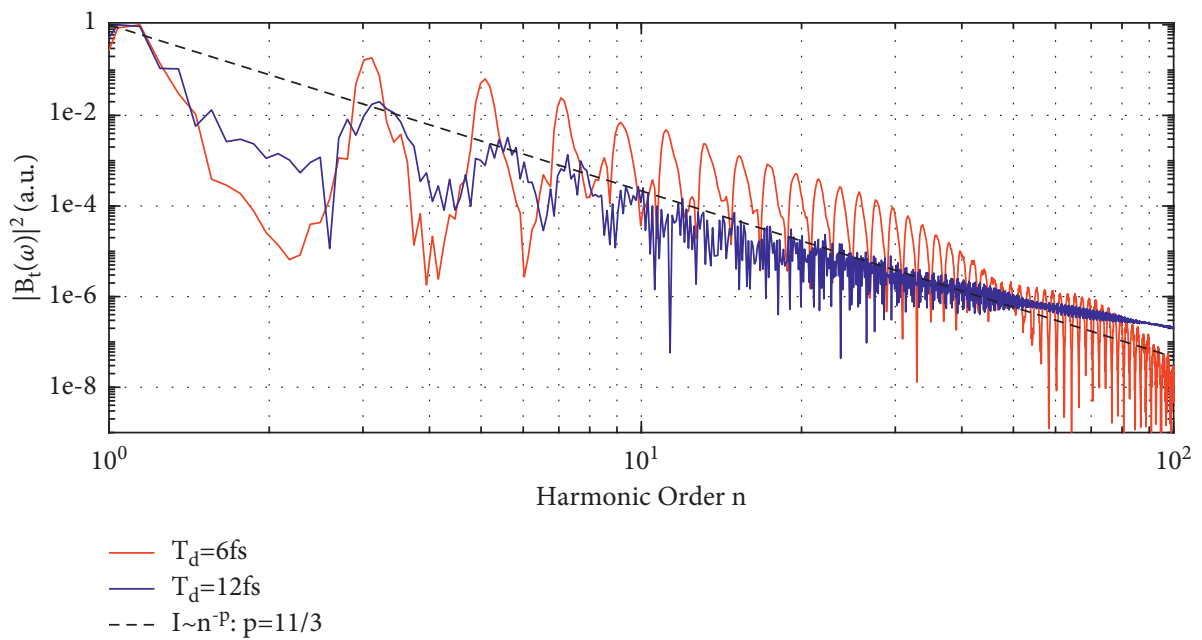

(a)

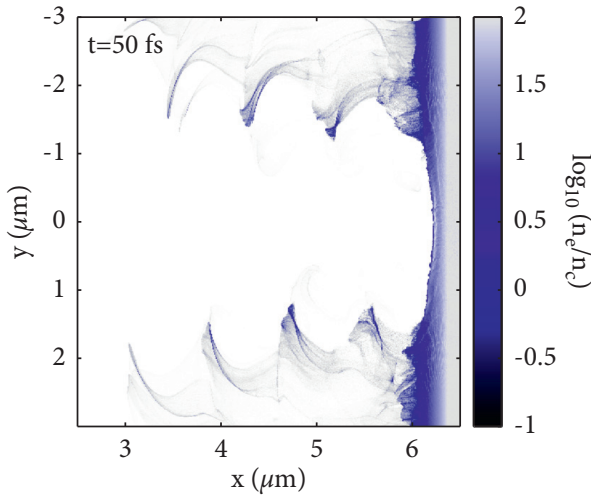

(b)

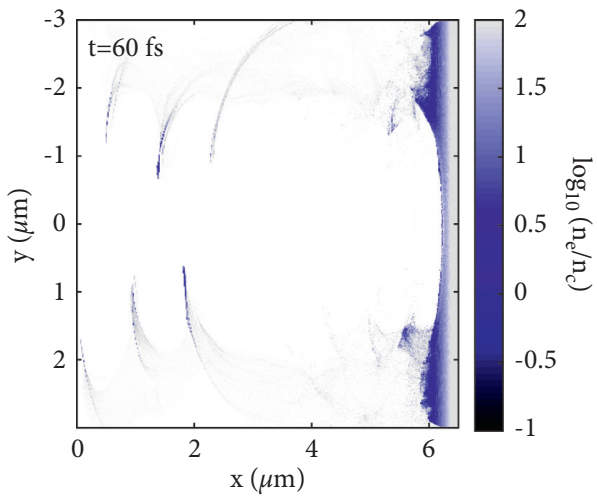

(c)

FIGURE 6: 2D PIC simulation. (a) Intensity boost and modulation of harmonic spectrum in the polarization gating scheme. The intensity of HHG with optimal time delay (red curve) can be increased by approximately 100 times when compared with the other nonoptimal time delay (blue curve). The other simulation parameters are $a=10, \tau=6 T_{0}, n_{e}=100 n_{c}, L s=0.2 \lambda_{L}$, and normal incidence. (b, c) Snapshots of electron density at different times when the time delay is $6 f_{s}$. The other simulation parameters are $a=10, \tau=6 T_{0}, n_{e}=100 n_{c}, L s=0.2 \lambda_{L}$, and normal incidence. 


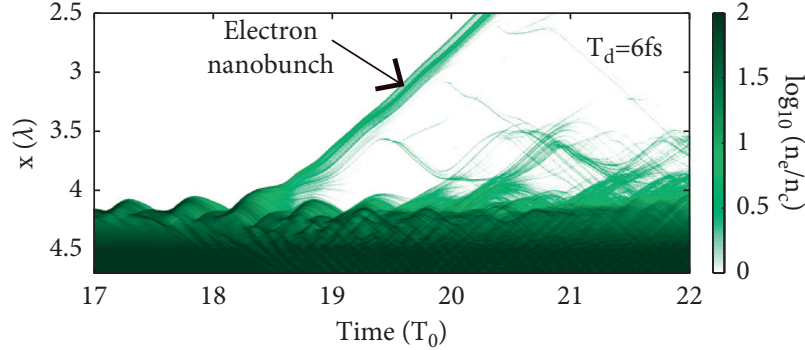

(a)

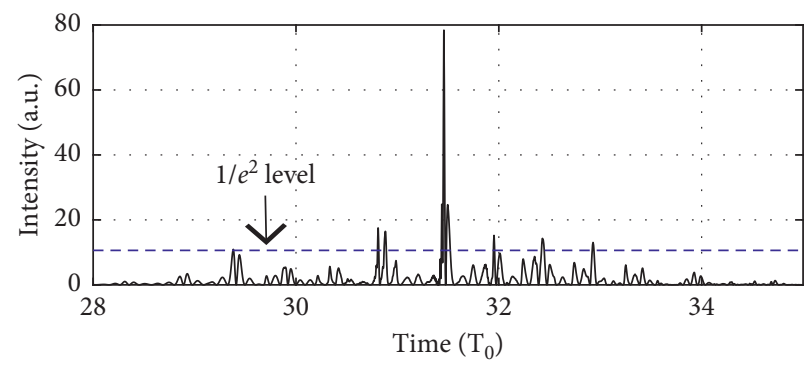

(c)

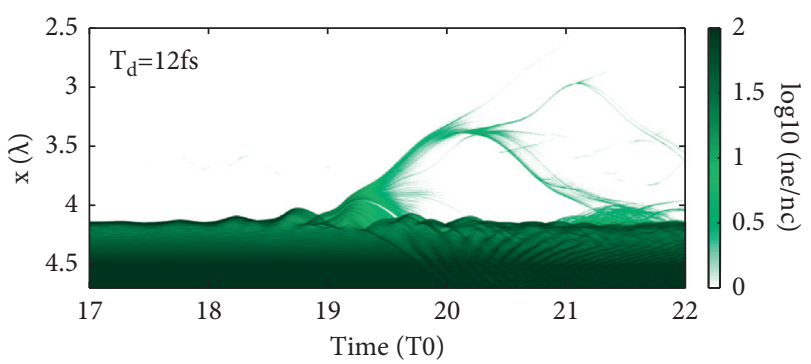

(b)

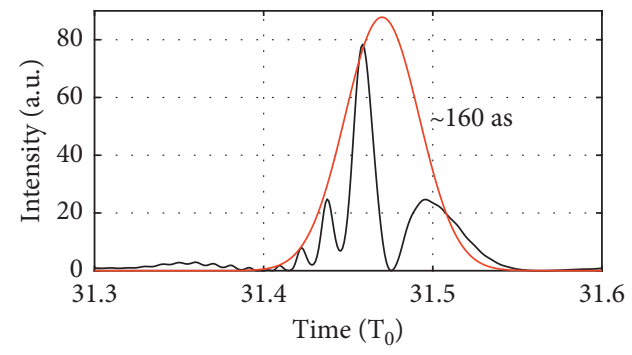

(d)

Figure 7: (a, b) The spatiotemporal evolution of electron density with delay $T_{d}=6 f s$ and $12 f s$, respectively. (c) Obtained radiation pulse intensity by applying a spectral filter to select the 30th to 100th harmonic orders at $T_{d}=6 f s$. (d) Zoomed view of a single pulse with envelope fitting (red). The other simulation parameters are $a=10, \tau=6 T_{0}, L_{s}=0.2 \lambda_{L}, n_{e}=100 n_{c}$, and normal incidence.

electrons move back and are accelerated to the maximum longitudinal velocity by the combined field of space-charge field and the laser field. This shows that the harmonic generation can be boosted at an optimal time delay. The efficient CSE can be restricted to occur only once near the cycle of the peak laser intensity. At $L_{s}=0.2 \lambda_{L}$ and $T_{d}=6 f s$, the attosecond pulse profile is shown in Figure $7(\mathrm{c})$, which is filtered from the reflected field in the interval $30<\omega /$ $\omega_{0}<100$. Figure $7(d)$ shows the zoomed single attosecond pulse with an envelope fitting (red), where the FWHM pulse duration is approximately 160 attoseconds. Its normalized intensity is boosted to $\sim 80$, corresponding to $1.7 \times 10^{20} \mathrm{~W} /$ $\mathrm{cm}^{2}$. These results show the potential of multicycle laser pulses to produce isolated and bright attosecond extreme ultraviolet and soft X-ray pulses.

The ability to generate intense high harmonics with polarization gating is particularly important because it shows that the relativistic surface high harmonics have not only higher conversion efficiency but also fewer strict requirements on the driving field, making the relativistic surface high harmonics combined with polarization gating a very promising source of bright attosecond extreme ultraviolet and soft X-ray pulses. The proposed scheme is robust and can soon be tested in experiments with petawatt-femtosecond lasers.

\section{Conclusions}

We have presented results from a large number of particlein-cell simulations, demonstrating that the polarization gating concept applied in relativistic surface high-harmonic generation can be used to overcome the limitation typically associated with the relatively long preplasma scale length. An ultra-dense electron bunch and a strong electrostatic potential are formed owing to the pondermotive force of the incident pulse, which results in a strong coherent synchrotron emission. We have demonstrated numerically that the intensity of high-harmonic generation from laser-plasma interaction can be enhanced while maintaining the same total laser energy. Specifically, we have shown that the time delay in the polarization gating concept is crucial for highharmonic generation. The intensity of high-harmonic generation with optimal time delay can be boosted approximately 100 times when compared with the other nonoptimal time delay. The scheme proposed here points to the bright attosecond extreme ultraviolet pulse generation driven by petawatt-class multicycle laser pulses.

\section{Data Availability}

The data used to support the findings of this study are available from the corresponding author upon request.

\section{Conflicts of Interest}

The authors declare that there are no conflicts of interest.

\section{Acknowledgments}

The authors would like to thank the National Supercomputing Center in Guangzhou (NSCC-GZ) for the computing source. This work was supported by the National Natural Science Foundation of China (grant nos. 11922412, 11874372, and 91950203), Strategic Priority Research Program (B) (grant no. XDB16), Key Research Program of 
Frontier Sciences, CAS (grant no. ZDBS-LY-SLH018), Youth Innovation Promotion Association of Chinese Academy of Sciences, China Postdoctoral Science Foundation (grant no. 2021M703329), and Shanghai Pilot Program for Basic Research-Chinese Academy of Science, Shanghai Branch.

\section{References}

[1] B. Dromey, M. Zepf, A. Gopal et al., "High harmonic generation in the relativistic limit," Nature Physics, vol. 2, no. 7, pp. 456-459, 2006.

[2] A. Pukhov, "X-rays in a flash," Nature Physics, vol. 2, no. 7, pp. 439-440, 2006.

[3] C. Thaury, F. Quéré, J.-P. Geindre et al., "Plasma mirrors for ultrahigh-intensity optics," Nature Physics, vol. 3, no. 6, pp. 424-429, 2007.

[4] Y. Nomura, R. Hörlein, P. Tzallas et al., "Attosecond phase locking of harmonics emitted from laser-produced plasmas," Nature Physics, vol. 5, no. 2, pp. 124-128, 2009.

[5] R. Hörlein, Y. Nomura, P. Tzallas et al., "Temporal characterization of attosecond pulses emitted from solid-density plasmas," New Journal of Physics, vol. 12, Article ID 043020, 2010.

[6] F. Quéré, C. Thaury, P. Monot et al., "Coherent wake emission of high-order harmonics from overdense plasmas," Physical Review Letters, vol. 96, Article ID 125004, 2006.

[7] F. Quéré, C. Thaury, J. P. Geindre, G. Bonnaud, P. Monot, and P. Martin, "Phase properties of laser high-order harmonics generated on plasma mirrors," Physical Review Letters, vol. 100, Article ID 095004, 2008.

[8] G. D. Tsakiris, K. Eidmann, J. Meyer-ter-Vehn, and F. Krausz, "Route to intense single attosecond pulses," New Journal of Physics, vol. 8, 2006.

[9] S. V. Bulanov, N. M. Naumova, and F. Pegoraro, "Interaction of an ultrashort, relativistically strong laser pulse with an overdense plasma," Physics of Plasmas, vol. 1, no. 3, pp. 745-757, 1994.

[10] T. Baeva, S. Gordienko, and A. Pukhov, "Theory of high-order harmonic generation in relativistic laser interaction with overdense plasma," Physical review. E, Statistical, nonlinear, and soft matter physics, vol. 74, Article ID 046404, 2006.

[11] B. Dromey, S. Kar, C. Bellei et al., "Bright multi-keV harmonic generation from relativistically oscillating plasma surfaces," Physical Review Letters, vol. 99, Article ID 085001, 2007.

[12] B. Dromey, D. Adams, R. Hörlein et al., "Diffraction-limited performance and focusing of high harmonics from relativistic plasmas," Nature Physics, vol. 5, no. 2, pp. 146-152, 2009.

[13] L. Plaja, L. Roso, K. Rzazewski, and M. Lewenstein, "Generation of attosecond pulse trains during the reflection of a very intense laser on a solid surface," Journal of the Optical Society of America B, vol. 15, no. 7, pp. 1904-1911, 1998.

[14] R. Lichters, J. Meyer-ter-Vehn, and A. Pukhov, "Short-pulse laser harmonics from oscillating plasma surfaces driven at relativistic intensity," Physics of Plasmas, vol. 3, no. 9, pp. 3425-3437, 1996.

[15] B. Dromey, S. Cousens, S. Rykovanov et al., "Coherent synchrotron emission in transmission from ultrathin relativistic laser plasmas," New Journal of Physics, vol. 15, 2013.

[16] B. Dromey, S. Rykovanov, M. Yeung et al., "Coherent synchrotron emission from electron nanobunches formed in relativistic laser-plasma interactions," Nature Physics, vol. 8, no. 11, pp. 804-808, 2012.

[17] A. Pukhov, D. An der Brugge, and I. Kostyukov, "Relativistic laser plasmas for electron acceleration and short wavelength radiation generation," Plasma Physics and Controlled Fusion, vol. 52, 2010.

[18] D. a. d. Brügge and A. Pukhov, "Enhanced relativistic harmonics by electron nanobunching," Physics of Plasmas, vol. 17, Article ID 033110, 2010.

[19] A. A. Gonoskov, A. V. Korzhimanov, A. V. Kim, M. Marklund, and A. M. Sergeev, "Ultrarelativistic nanoplasmonics as a route towards extreme-intensity attosecond pulses," Physical review. E, Statistical, Nonlinear, and Soft Matter Physics, vol. 84, Article ID 046403, 2011.

[20] S. Kahaly, S. Monchocé, H. Vincenti et al., "Direct observation of density-gradient effects in harmonic generation from plasma mirrors," Physical Review Letters, vol. 110, no. 17, Article ID 175001, 2013.

[21] L. Chopineau, A. Leblanc, G. Blaclard et al., "Identification of coupling mechanisms between ultraintense laser light and dense plasmas," Phys.Rev.X, vol. 9, Article ID 011050, 2019.

[22] Z. Zhang, F. Wu, J. Hu et al., "The laser beamline in SULF facility," High Power Laser Science and Engineering, vol. 8, p. e4, 2020.

[23] S. G. Rykovanov, M. Geissler, J. Meyer-ter-Vehn, and G. D. Tsakiris, "Intense single attosecond pulses from surface harmonics using the polarization gating technique," New Journal of Physics, vol. 10, no. 2, Article ID 025025, 2008.

[24] T. Baeva, S. Gordienko, and A. Pukhov, "Relativistic plasma control for single attosecond $\mathrm{x}$-ray burst generation," Physical review. E, Statistical, nonlinear, and soft matter physics, vol. 74, Article ID 065401, 2006.

[25] M. Yeung, J. Bierbach, E. Eckner et al., "Noncollinear polarization gating of attosecond pulse trains in the relativistic regime," Physical Review Letters, vol. 115, Article ID 193903, 2015.

[26] P. B. Corkum, N. H. Burnett, and M. Y. Ivanov, "Subfemtosecond pulses," Optics Letters, vol. 19, no. 22, pp. 1870-1872, 1994.

[27] V. T. Platonenko and V. V. Strelkov, "Single attosecond soft$\mathrm{x}$-ray pulse generated with a limited laser beam," Journal of the Optical Society of America B, vol. 16, no. 3, pp. 435-440, 1999.

[28] T. D. Arber, K. Bennett, C. S. Brady et al., "Contemporary particle-in-cell approach to laser-plasma modelling," Plasma Physics and Controlled Fusion, vol. 57, no. 11, Article ID 113001, 2015.

[29] F. Quéré and H. Vincenti, "Reflecting petawatt lasers off relativistic plasma mirrors: a realistic path to the Schwinger limit," High Power Laser Sci. Eng.vol. 9, 2021.

[30] J. Yan, N. Huang, H. Deng, B. Liu, D. Wang, and Z. Zhao, "First observation of laser-beam interaction in a dipole magnet," Advanced Photonics, vol. 3, Article ID 045003, 2021. 